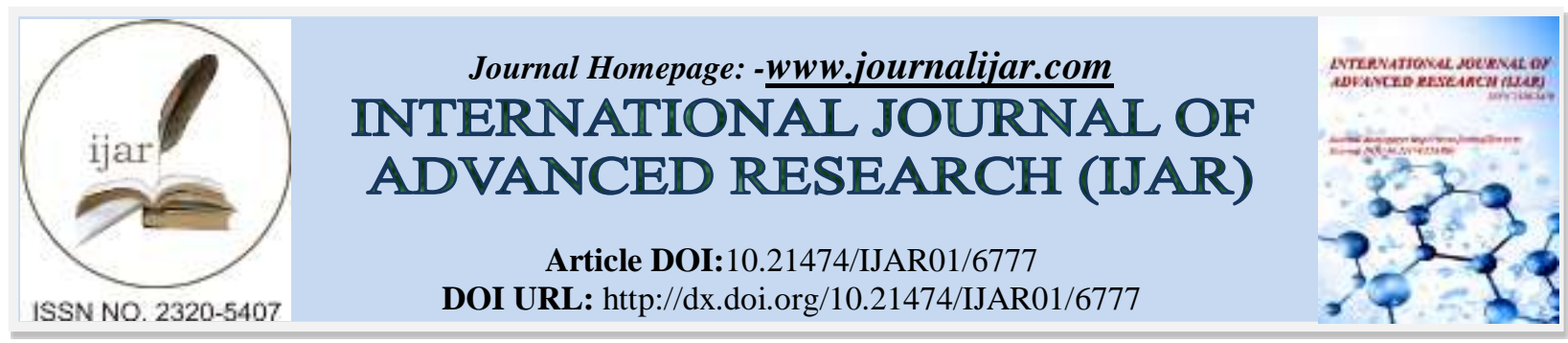

RESEARCH ARTICLE

\title{
ASSESSMENT OF KNOWLEDGE, ATTITUDE AND PRACTICES OF MCH COMPONENTS AMONG YOUNG MOTHERS.
}

B. Varalakshmi.

\section{Manuscript Info}

Manuscript History

Received: 18 January 2018

Final Accepted: 20 February 2018

Published: March 2018

\section{Abstract}

A descriptive study was undertaken to Assess the mothers knowledge, attitudes and practices of $\mathrm{MCH}$ components at selected urban community, Sriramnagar, UPHC, Hyderabad, Telangana.

A sample of 500 mothers was chosen through purposive convenient sampling technique. A structured interview questionnaire was used to collect the data. Findings revealed that majority of mothers scored above average and average knowledge levels. Their practices are very poor that is $80 \%$ are below average.

Objectives:

1. To assess the mothers knowledge, attitudes and practices regarding antenatal care.

2. To examine the attitudes of mothers regarding contraceptive methods.

3. To identify/observe the practices about breast feeding and Immunization.

4. To study the association between KAP on MCH components and socioeconomic and demographic variables

Copy Right, IJAR, 2018,. All rights reserved.

\section{Introduction:-}

Health is the precious possession of all human beings as it is asset for an individual and community as well. Maternal and child health is recognized as one of the significant components of family welfare. Health of both mother and children is a matter of public health concern because of several reasons.

Women in child bearing age and growing infants and children under 15 years of age together form a large section of our society $(19 \%+40 \%=59 \%)$, and such majority of public health services would be consumed by them. They are the more dependent and vulnerable members of the society who are at a high risk of morbidity and mortality.

In India 125000(46 per 100000 live births) women die due to pregnancy and child birth related causes. About 1.8 million (74per 1000 live births) infants and 2.65 million (109 per 1000 live births) under five children die every year. Most of these deaths are preventable. Hence a greater priority is given by public health personnel to maternal and child health services to reduce maternal and child morbidity and mortality and improve community health.

\section{Methodology:-}

Research design, sample \& setting:-

Descriptive study was undertaken to assess the mothers knowledge regarding $\mathrm{MCH}$ component (antenatal care, breast feeding, immunization and family planning). A purposive convenient sampling technique was used to select 
the sample for the study. 500 mothers was selected from two urban slums, those are Siramnagar and Jawarnagar community. Mothers who are in reproductive age group (15 to 45 years) and having at least one living child, the mother who were able to speak and understand Telugu, English and Hindi. The present study was conducted in Sriramnagar and Jawaharnagar, Urban community, Hyderabad. This is situated $6 \mathrm{~km}$ away from NIMS hospital, and attached to UPHC, Sriramnagar. In this community areas belongs to different religion i.e. Hindu, Muslim and Christian.

\section{Instrument:-}

A structured interview schedule was used to collect the data.

\section{Data collection procedure:-}

The period of data was collected from 13.03.2015 to 15.05.2015, before starting data collection permissions was obtained from DM\&HO, Hyderabad, District, Telangana, and medical officer of Sriramnagar, UPHC.

\section{Data analysis:-}

Descriptive and inferential statistics were used to analyse the data, correlations was used to determine the relationship between mothers Knowledge, Attitudes and Practices with their demographic variables.

\section{Findings:-}

Table-1:- Frequency Percentage Distribution of Socio, Economic \& Demographic Variables

\begin{tabular}{|c|c|c|c|c|c|}
\hline \multirow[t]{2}{*}{ S.No. } & \multirow[t]{2}{*}{ Demographic Variables } & \multicolumn{2}{|c|}{ Sriram Nagar } & \multicolumn{2}{|c|}{ Jawahar Nagar } \\
\hline & & $\mathbf{F}$ & P \% & $\mathbf{F}$ & P \% \\
\hline 1 & $\begin{array}{l}\text { RESIDENCE } \\
0-1 \text { year } \\
1-5 \text { years } \\
>5 \text { years }\end{array}$ & $\begin{array}{c}23 \\
96 \\
131 \\
\end{array}$ & $\begin{array}{c}9.2 \\
38.4 \\
52.4 \\
\end{array}$ & $\begin{array}{c}30 \\
68 \\
152 \\
\end{array}$ & $\begin{array}{l}12.0 \\
27.2 \\
60.8 \\
\end{array}$ \\
\hline 2 & $\begin{array}{l}\text { AGE } \\
21-25 \text { years } \\
26-30 \text { years }\end{array}$ & $\begin{array}{l}120 \\
130\end{array}$ & $\begin{array}{l}48.0 \\
52.0\end{array}$ & $\begin{array}{c}167 \\
83\end{array}$ & $\begin{array}{r}66.8 \\
33.2 \\
\end{array}$ \\
\hline 3 & $\begin{array}{l}\text { RELIGION } \\
\text { Hindu } \\
\text { Christian } \\
\text { Muslims } \\
\text { Others } \\
\end{array}$ & $\begin{array}{c}126 \\
45 \\
74 \\
5 \\
\end{array}$ & $\begin{array}{c}50.4 \\
18.0 \\
29.6 \\
2.0 \\
\end{array}$ & $\begin{array}{c}122 \\
35 \\
84 \\
9\end{array}$ & $\begin{array}{c}48.8 \\
14.0 \\
33.6 \\
3.6 \\
\end{array}$ \\
\hline 4 & $\begin{array}{l}\text { EDUCATION } \\
\text { Illiterate } \\
\text { Preprimary } \\
\text { Upper Primary } \\
\text { Intermediate } \\
\text { Degree } \\
\end{array}$ & $\begin{array}{l}37 \\
55 \\
73 \\
48 \\
37\end{array}$ & $\begin{array}{l}14.8 \\
22.0 \\
29.2 \\
19.2 \\
14.8\end{array}$ & $\begin{array}{c}37 \\
27 \\
107 \\
20 \\
59 \\
\end{array}$ & $\begin{array}{c}14.8 \\
10.8 \\
42.8 \\
8.0 \\
23.6\end{array}$ \\
\hline 5 & $\begin{array}{l}\text { OCCUPATION } \\
\text { House Wife } \\
\text { Employee } \\
\text { Daily Wage } \\
\text { Business } \\
\end{array}$ & $\begin{array}{c}222 \\
18 \\
10 \\
0.0 \\
\end{array}$ & $\begin{array}{c}88.8 \\
7.2 \\
4.0 \\
0.0 \\
\end{array}$ & $\begin{array}{c}220 \\
14 \\
11 \\
5 \\
\end{array}$ & $\begin{array}{c}88.0 \\
5.6 \\
4.4 \\
2.0 \\
\end{array}$ \\
\hline 6 & $\begin{array}{l}\text { INDEPENDENT INCOME } \\
\text { No Income } \\
\text { Rs. } 5000-6000 /- \\
\text { Rs. } 6001-7000 /- \\
\text { Rs. } 7001-8000 /- \\
\text { Rs. } 8001-10000 /- \\
\end{array}$ & $\begin{array}{c}223 \\
10 \\
7 \\
5 \\
5\end{array}$ & $\begin{array}{c}89.2 \\
4.0 \\
2.8 \\
2.0 \\
2.0\end{array}$ & $\begin{array}{c}213 \\
19 \\
2 \\
3 \\
13 \\
\end{array}$ & $\begin{array}{c}85.2 \\
73.6 \\
8 \\
1.2 \\
5.2\end{array}$ \\
\hline 7 & $\begin{array}{l}\text { FAMILY INCOME } \\
\text { Rs. } 10000-15000 /- \\
\text { Rs. } 15001-20000 /- \\
\text { Rs. } 20001-25000 /-\end{array}$ & $\begin{array}{c}163 \\
49 \\
30\end{array}$ & $\begin{array}{l}65.2 \\
19.6 \\
12.0 \\
\end{array}$ & $\begin{array}{c}192 \\
19 \\
8 \\
\end{array}$ & $\begin{array}{c}76.8 \\
7.6 \\
3.2\end{array}$ \\
\hline
\end{tabular}




\begin{tabular}{|c|c|c|c|c|c|}
\hline & $>25000 /-$ & 8 & 3.2 & 31 & 12.4 \\
\hline 8 & $\begin{array}{l}\text { NUMBER OF CHILDREN } \\
\text { One } \\
\text { Two } \\
\text { Three } \\
\text { Four }\end{array}$ & $\begin{array}{c}90 \\
135 \\
18 \\
7\end{array}$ & $\begin{array}{c}36.0 \\
54.0 \\
7.2 \\
2.8\end{array}$ & $\begin{array}{c}101 \\
100 \\
48 \\
1\end{array}$ & $\begin{array}{l}40.4 \\
40.0 \\
19.2 \\
0.4\end{array}$ \\
\hline 9 & $\begin{array}{l}\text { TYPE OF FAMILY } \\
\text { Nuclear Family } \\
\text { Joint Family }\end{array}$ & $\begin{array}{c}194 \\
56\end{array}$ & $\begin{array}{l}77.6 \\
22.4\end{array}$ & $\begin{array}{l}129 \\
121\end{array}$ & $\begin{array}{l}51.6 \\
48.4\end{array}$ \\
\hline 10 & $\begin{array}{l}\text { TYPE OF HOUSE } \\
\text { Pucca } \\
\text { Kutcha } \\
\text { Semihut }\end{array}$ & $\begin{array}{c}213 \\
30 \\
7\end{array}$ & $\begin{array}{l}85.2 \\
12.0 \\
2.8\end{array}$ & $\begin{array}{c}173 \\
75 \\
2\end{array}$ & $\begin{array}{c}69.2 \\
30.0 \\
8.0\end{array}$ \\
\hline 11 & $\begin{array}{l}\text { RENTED / OWN HOUSE } \\
\text { Rent House } \\
\text { Own House }\end{array}$ & $\begin{array}{l}150 \\
100\end{array}$ & $\begin{array}{l}60.0 \\
40.0\end{array}$ & $\begin{array}{c}180 \\
70\end{array}$ & $\begin{array}{l}72.0 \\
28.0\end{array}$ \\
\hline 12 & $\begin{array}{l}\text { ELECTRICITY } \\
\text { No } \\
\text { Yes }\end{array}$ & $\begin{array}{c}6 \\
244 \\
\end{array}$ & $\begin{array}{c}2.4 \\
97.6 \\
\end{array}$ & $\begin{array}{c}5 \\
245 \\
\end{array}$ & $\begin{array}{r}2.0 \\
98.0 \\
\end{array}$ \\
\hline 13 & $\begin{array}{l}\text { ROOMS IN HOUSE } \\
\text { One } \\
\text { Two } \\
\text { Two }\end{array}$ & $\begin{array}{l}87 \\
79 \\
84\end{array}$ & $\begin{array}{l}34.8 \\
31.6 \\
33.6\end{array}$ & $\begin{array}{l}95 \\
83 \\
72\end{array}$ & $\begin{array}{l}38.0 \\
33.2 \\
28.8\end{array}$ \\
\hline 14 & $\begin{array}{l}\text { SEPARATE BED ROOM } \\
\text { No } \\
\text { Yes }\end{array}$ & $\begin{array}{l}140 \\
110\end{array}$ & $\begin{array}{l}56.0 \\
44.0\end{array}$ & $\begin{array}{l}146 \\
104\end{array}$ & $\begin{array}{l}58.4 \\
41.6\end{array}$ \\
\hline 15 & $\begin{array}{l}\text { PRESENT OF SANITARY LATRINE } \\
\text { No } \\
\text { Yes }\end{array}$ & $\begin{array}{c}2 \\
248 \\
\end{array}$ & $\begin{array}{c}0.8 \\
99.2 \\
\end{array}$ & $\begin{array}{c}4 \\
246 \\
\end{array}$ & $\begin{array}{c}1.6 \\
98.4\end{array}$ \\
\hline 16 & $\begin{array}{l}\text { DISPOSAL OF SANITARY NAPKIN } \\
\text { Burning } \\
\text { Dumping } \\
\text { Rapped in a paper } \\
\text { Put in dustbin }\end{array}$ & $\begin{array}{c}23 \\
11 \\
216\end{array}$ & $\begin{array}{c}9.2 \\
4.4 \\
33.6\end{array}$ & $\begin{array}{c}19 \\
18 \\
213\end{array}$ & $\begin{array}{r}7.6 \\
7.2 \\
85.2\end{array}$ \\
\hline 17 & $\begin{array}{l}\text { MATERIAL USED } \\
\text { Cloth } \\
\text { Sanitary Napkin }\end{array}$ & $\begin{array}{c}44 \\
206 \\
\end{array}$ & $\begin{array}{l}17.6 \\
82.4\end{array}$ & $\begin{array}{c}56 \\
194 \\
\end{array}$ & $\begin{array}{l}22.4 \\
77.6 \\
\end{array}$ \\
\hline 18 & $\begin{array}{l}\text { SOURCE OF WATER FOR } \\
\text { DRINKING } \\
\text { Municipal Public Tab } \\
\text { Municipal Private Tap } \\
\text { Bore Water }\end{array}$ & $\begin{array}{c}64 \\
183 \\
3\end{array}$ & $\begin{array}{c}25.6 \\
73.2 \\
1.2\end{array}$ & $\begin{array}{c}57 \\
192 \\
1\end{array}$ & $\begin{array}{c}22.8 \\
76.8 \\
0.4\end{array}$ \\
\hline
\end{tabular}

\section{Discussion:-}

Mother's Knowledge, Attitudes and Practices in Sriramnagar:-

The findings revealed that, there is a association between mothers knowledge on $\mathrm{MCH}$ components with their religion, education, occupation, family income, type of house, number of rooms in the house, disposal method of sanitary napkins and material used for sanitary napkins.

Association of mothers attitudes on MCH components with their demographic variables like number of children and type of house. 
Association of mothers practices on $\mathrm{MCH}$ components with their demographic variables such as age, religion, education, occupation, independent income, disposal method of sanitary napkins and source of getting drinking water etc.

\section{Mothers Knowledge, Attitudes and Practices in Jawaharnagar:-}

The findings revealed that, there is a association between mothers knowledge on $\mathrm{MCH}$ components with their residence, age, religion, education, type of family, presence of sanitary latrine, disposal of sanitary napkin and material used for sanitary napkin.

Association of mothers attitudes on $\mathrm{MCH}$ components with their demographic variables like age, education, occupation, independent income, type of house and rent/ own house.

Association of mothers practices on $\mathrm{MCH}$ components with their demographic variables like age, education, occupation, independent income, number of rooms in the house and material used for sanitary napkins.

Table -2:- Mothers total Knowledge, Attitudes and Practices on MCH components.

\begin{tabular}{|l|c|c|c|c|}
\hline Variables & \multicolumn{2}{|c|}{$\begin{array}{c}\text { Below average } \\
(<\mathbf{3 3 . 3 \%})\end{array}$} & $\begin{array}{c}\text { Average } \\
(\mathbf{3 3 . 4} \text { to 66.6\%) }\end{array}$ & $\begin{array}{c}\text { Above average } \\
(>\mathbf{6 6 . 6 \%})\end{array}$ \\
\hline \multirow{2}{*}{ Knowledge } & $\mathrm{F}$ & 7 & 216 & 277 \\
\cline { 2 - 5 } & $\%$ & $1.4 \%$ & $43.2 \%$ & $55.4 \%$ \\
\hline \multirow{2}{*}{ Attitudes } & $\mathrm{F}$ & 18 & 415 & 67 \\
\cline { 2 - 5 } & $\%$ & $3.6 \%$ & $83.0 \%$ & $33.4 \%$ \\
\hline \multirow{2}{*}{ Practices } & $\mathrm{f}$ & 394 & 73 & $6.6 \%$ \\
\cline { 2 - 5 } & $\%$ & $78.8 \%$ & $14.6 \%$ & 33 \\
\hline
\end{tabular}

Above table-2 reveals that, mothers knowledge scores, out of 500 mothers only 7 (1.4\%) were having below average, 216(43.2\%) were having average, and 277 (55.4\%) were having above average knowledge scores.

Mothers attitudes scores regarding $\mathrm{MCH}$ components, out of 500 subjects 18 (3.6\%) were scored below average, $415(83.0 \%)$ scored average and $67(13.4 \%)$ were scored above average scores.

Mothers practice scores on $\mathrm{MCH}$ components, out of 500 respondents 394(78.8\%) were got below average, 73 $(14.6 \%)$ were got average and only $33(6.6 \%)$ were got above average scores.

\section{Conclusion:-}

Conclusions were drawn on the basis of the findings of the study majority of the mothers $277(55.4 \%)$ were having above average knowledge on $\mathrm{MCH}$ components and attitudes of the mothers $415(83.0 \%)$ were scored average and practices of the mothers $33(6.6 \%)$ were scored above average, but $394(78.8 \%)$ of the mothers got below average levels those who were not practising.

\section{References:-}

1. Park. J.E. and K.Park 1991, Text book of preventive and social Medicine, Jabalpur/S Banarasi das Bhannot Publishers.

2. Dutta.D.C.Text book of Obstetrical and perinatology including contraception, 4 th edition, Central book agencies publications.

3. Marlow.R.Dorothy and redding A.Barhara 1988, Text book of Pediatrics Nursing, Philadelphia, W.B.Sunders company.

4. K.K.Gulani 2005, Community Health Nursing principles and practices, Delhi, Kumar publishing house, $1^{\text {st }}$ edition, pg.no. 299-336.

5. B.T.Basavanthappa, 1988, Community Health Nursing, New Delhi, Jaypee Brothers medical publishers (Pvt) limited. $1^{\text {st }}$ edition. Pg. no. 317-340.

6. B.T.Basavanthappa, 1988, Nursing Research. New Delhi. Jaypee Brothers medical publishers (Pvt) limited. $1^{\text {st }}$ edition. 


\section{Journals:-}

1. DasD.K.et al, 1995, "Knowledge and attitudes of the Bangladesh Rural regarding breast feeding and weaning, Indian Journal of Paediatrics", vol.152, pg no. 889-892.

2. Barton.S.J. 2001, "Infant feeding practices of low income rural mothers", American Journal of Maternal child nursing. March-April. 26. Pg. no. 93-97.

3. Shweta Dabal and S.L Malik 2002. "Demographic study of Gujjare of Delhi, knowledge, attitudes and practices of family planning" Journal of human ecology 16(4), pg. no. 231-237(2004).

4. Rajesh Reddy S.K.C.Premajavan, K.A. Narayan and Akshaya Kumar Mishra, 2002. "Rapid appraisal of knowledge, attitudes and practices related to family planning methods among men within 5 years of marital life". Indian Journal on preventive and social medicine, Vol.34. No.1\&2, pg. no.63-67.

5. R.S.Sharma, M.Rajyalakshmi and D.Antony JeyRaj 2001, "study on current status of fertility control methods in India". Journal of Bio-sciences Vol.26, No.4, pg. no.391-405.

6. Padma Mohan, Asha Kamath and BS Sajjen.2003, "Fertility pattern and family planning practices in a rural area in Dakshina Kannada".Indian Journal of Community Medicine,Vol.28. Jan-March 2003, pg.no. 15-18.

7. Knowledge, attitudes and practices on antenatal care among Orang asli women in Jempol, Negeri Sembilen, faculty of medicine and health sciences, Malaysian Journal of public health medicine, 2011.Vol.11 (2) 13-21. 